\title{
Microforming of Stainless Steel Miniature Pump by Additive Sheet-Manufacturing
}

\author{
Tatsuhiko Aizawa ${ }^{1, *}$ and Tomomi Shiratori ${ }^{2}$ \\ ${ }^{1}$ Surface Engineering Design Laboratory, Shibaura Institute of Technology, Tokyo 144-0045, Japan \\ ${ }^{2}$ Komatsu-Seiki-Kosakusho, Co., Ltd., Suwa 392-0012, Japan
}

\begin{abstract}
Micro-pump had an integrated structure including the valves, the reservoir, the actuating plates and so forth as designed for each application. Various liquid as well as viscous media popped in and out through this unit; each element must have sufficient strength and toughness as well as well-defined accuracy in geometry and dimension. In particular, high leak proof is the highest requirement for this micropump in working at the medical operation, at the drug delivery and at the blood transportation. The additive sheet-manufacturing was proposed to make micro-forming of these miniature mechanical elements, devices and systems. This process consisted of three steps. Fist, the original CAD data for a micro-pump were sliced into an assembly of geometric models for each perforated and embossed sheet form. In second, a pair of miniature punch and die was fabricated by the plasma printing process after each geometric model. Then, each bare sheet element was micropierced and micro-embossed to transform each CAD-model to each shaped stainless steel sheet element. Finally, the assembly of sheet elements was plasma surface-activated and integrated into the tailored micro-pump by micro-joining. This top-to-down and down-to-top methodology in the above is discussed for further improvement of additive sheet-manufacturing. [doi:10.2320/matertrans.MT-ML2019007]
\end{abstract}

(Received July 31, 2019; Accepted September 30, 2019; Published December 6, 2019)

Keywords: digital manufacturing, plasma printing, micro die set, CNC micropiercing, plasma surface activation, low temperature diffusion bonding, micropump, stainless steel

\section{Introduction}

A pump works as a mechanical element to drive the liquid and gas mass transfer from one end to the other with the controlled flow rate and without negative pressure. ${ }^{1)}$ Irrespective of its size and shape, this positive displacement pump consists of four functionally structured parts in principle ${ }^{2)}$ e.g., the inlet and outlet channels in connection to the outer system, the valves to be working alternatively for sagging and sucking the liquid and gas media, the reservoir to control the flow rate and the driving unit. Many types of pumps are utilized with dependence on the media to be transferred by them as well as their size and the flow rate. The cast iron pump is used for mass transfer of the pressurized water solutions and gasses. ${ }^{3)}$ The machined and weld pump is utilized for circulation of liquid solutions and gasses; ${ }^{4)}$ in particular, an infusion pump is widely employed as a circulator of bloods, medicines, nutrients and so forth. ${ }^{5)}$ Among the pumps for medical use, the specially programmed pump after protocol by doctors is used in medical operation to preserve the well-conditioned state of operated body. ${ }^{6}$ Even in the agricultural engineering, the miniature pump is expected to periodically supply the nutrition to each plant, vegetable and fruit. ${ }^{7}$ Irrespective of its applications, this miniature pump must have sufficient strength and toughness enough to establish the leak proof even in the down-sized micro-pumping system.

This micro-pump was first proposed as an application of silicon technology in MEMS to fabricate the miniature mechanical element. ${ }^{8}$ ) Although its demonstration was attractive to reduce the element size, the silicon based system is too fragile to be used in practical situation. A metallic micro-pump is expected to put the miniature pumping unit into practice; there are three approaches in fabrication of metallic micro-pumps.
An additive manufacturing ${ }^{9,10)}$ is the first powerful tool to shape three dimensional parts, members and components in metals and plastics. In particular, this approach provides an integrated process to fabricate the optimally structured parts in the aerospace engineering from metallic and alloyed powders. Titanium alloy powders were utilized to make a micropump in trial. ${ }^{11)}$ In second, the friction sheet welding ${ }^{12)}$ has been utilized to fabricate a complex shaped aluminum alloy component by joining of parts. Those two manufacturing processes in the above stand on the melting of starting materials; the final products have heat-affected zones with low toughness. Authors proposed the additive sheetmanufacturing to fabricate the fine orifice plates with the shaped through-holes ${ }^{13}$ ) as well as the micro-pump as a precisely integrated assembly of segmented stainless steel sheets. ${ }^{14,15)}$

In the present paper, this additive sheet-manufacturing process is described and reconsidered as an effective tool of micro-forming. First, its top-to-down and down-to-top streams are explained to make geometric data processing in digital from the final product down to the constituent sheets and to integrate them up to the final product. In particular, four hardware technologies are stated as a micro-forming tool with orientation to digital processing; e.g., the plasma printing to fabricate the tailored miniature punch and die, the $\mathrm{CNC}$ stamping to make fine micro-piercing and microembossing into metallic sheets, the plasma surface activation to clean each sheet surface and the warm and hot joining by the intelligent induction heating. A micro-pump is employed as a targeting product to explain the above four steps in this additive sheet-manufacturing. In particular, optimization of plasma conditions for efficient surface activation is discussed together with search for homogeneous heating of assembly by induction heating. 


\begin{tabular}{|l|l|}
\hline Product Design of Micro-Pump \\
\hline Slicing of CAD data to shaping sheet & $\begin{array}{l}\text { Top-to-Bottom } \\
\text { Procedure } \\
\text { from Product } \\
\text { to Sheets }\end{array}$ \\
\hline Plasma Printing of Dies to Shape Each Sheet & \begin{tabular}{l} 
Down-to-Top \\
\hline Plasma Surface Activation of Sheet Surfaces
\end{tabular} \\
\hline Precise Micro-Joining into Micro-Pump & $\begin{array}{l}\text { Docedure } \\
\text { from sheets } \\
\text { to product }\end{array}$ \\
\hline
\end{tabular}

Fig. 1 Procedure of additive sheet-manufacturing to fabricate the micropump.

\section{Experimental Procedure}

\subsection{Data-driven processing}

The additive sheet-manufacturing has top-to-down and down-to-top streams as depicted in Fig. 1. In the former process, the original CAD data $(\mathrm{S})$ for product is sliced into an assembly of geometric model for each constituent segment $\{\mathrm{Si}\}$; e.g., $\mathrm{S}=\mathrm{U} \mathrm{Si}$ for $1<\mathrm{i}<\mathrm{n}$. In the present additivesheet manufacturing, this segment becomes a stainless steel sheet with the thickness of 10 to $50 \mu \mathrm{m}$. Each sheet has a functional geometry as a part of micro-pump. A pair of micro-punch and core-die is fabricated by the plasma printing to accommodate each functional geometry into each sheet through fine micro-piercing and micro-embossing. In the latter process, the whole top and bottom surfaces of shaped stainless steel element sheets are plasma activated to reduce the oxide passive film thickness enough to lower the diffusion bonding temperature. After this plasma surface activation, the assembly of sheets is micro-joined at lower holding temperature than $1000 \mathrm{~K}$ to be free from thermal distortion and heat affected zones and for shorter takt time toward mass production of miniature mechanical elements.

\subsection{Plasma printing}

In the former top-to-down approach in Fig. 1, the while CAD data for micro-pump is sliced to a segment data $\{S i\}$ for each i-th sheet with its functional geometry. The geometric model $\{P i\}$ is edited by subtraction of this segment data from the whole $\mathrm{CAD}$ data $\{W i\}$ for each i-th sheet; e.g., $P i=$ $\mathrm{Wi}-\mathrm{Si}$. Then, this $P i$ has one-to-one correspondence to a part of sheet to be pierced or embossed stamping. At the first step in the top-to-down metal forming, a screen film is produced in order that the micropattern on the screen corresponds to an exclusively negative pattern to this $\mathrm{Pi}$ after 16, 17). Hence, $S i$ is micro-patterned onto the stainless steel die substrate by the screen printing; $P i$ corresponds to an unprinted surface as a negative micro-pattern to Si. Since the unprinted surface of stainless steel substrate is selectively nitrided and hardened by plasma nitriding, the unprinted substrate surface with $P i$ is nitrided and hardened, but the printed surface with $S i$ is not nitrided and not hardened. This printed part is removed from the substrate by sand-blasting; the substrate with $P i$ becomes a head of piercing and embossing punch to actually subtract the part of sheet with
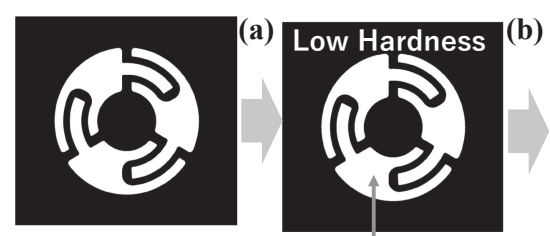

High Hardness



Fig. 2 A typical procedure of plasma printing to fabricate the micro-punch for micro-piercing each bare stainless sheet. (a) Screen-printing the tailored geometry of sliced product, (b) selective plasma nitriding at $673 \mathrm{~K}$ for $14.4 \mathrm{ks}$, and (c) blasting to remove the printed parts.

$P i$ from each i-th sheet and to fabricate the functional sheet with $S i$ for $1<i<n$.

Let us explain the above procedure in detail through the case study to fabricate the piercing punch to form a valve with a circular pendulum and three S-shaped springs into the stainless sheet. In this case, $\mathrm{Si}$ becomes a geometric model which is directly printed onto the bare stainless steel sheet in Fig. 2(a). The unprinted surface is plasma nitrided to have higher hardness than the blasting media selectively in Fig. 2(b). Finally, its printed part is removed in Fig. 2(c) by sand-blasting. In the following experiments, a screen printing system (Newlong, Co., Ltd.) was used for printing the mask pattern in Fig. 2(a). The low temperature plasma nitriding system ${ }^{18)}$ was also employed to make selective nitrogen supersaturation at $673 \mathrm{~K}$ for $14.4 \mathrm{ks}$ in Fig. 2(b). The sand-blasting machine (Fuji-Seisakusho, Co., Ltd.) was utilized for mechanical blasting with use of fine silica particles in Fig. 2(c).

\subsection{Micro-shaping of sheet elements}

The pair of micro-punch and core-die was set into a cassette die for micro-piercing as shown in Fig. 3(a). This die-set was fixed into the upper and lower bolsters in the CNC micro-stamper, as depicted in Fig. 3(b). In the following experiments, the micro-pump CAD model was sliced into eight elements. Each stainless steel sheet was micro-pierced to have perforates in correspondence to the sliced CAD data. Eight shaped sheet elements were stacked into an assembly of elements for plasma surface activation.

\subsection{Plasma surface activation}

Every surface in the assembly of sheet elements was activated and cleaned by the argon-hydrogen plasmas under the conditions where the RF (Radio-Frequency)-voltage was $150 \mathrm{~V}$, the DC (Direct Current)-bias voltage, $-100 \mathrm{~V}$ and the pressure, $80 \mathrm{~Pa}$. Figure 4(a) shows the micro-joining system including the surface activation unit and the hot joining unit. As depicted in Fig. 4(b), every top and bottom sheet surface was subjected to plasma irradiation.

\subsection{Precise micro-joining for integration}

The surface activated sheet assembly was restacked and uniaxially loaded at the specified temperature for diffusion bonding in Fig. 4(a). In the following experiments, the holding temperature and applied pressure are varied to describe the joinability between the surface activated stainless steel sheets. 


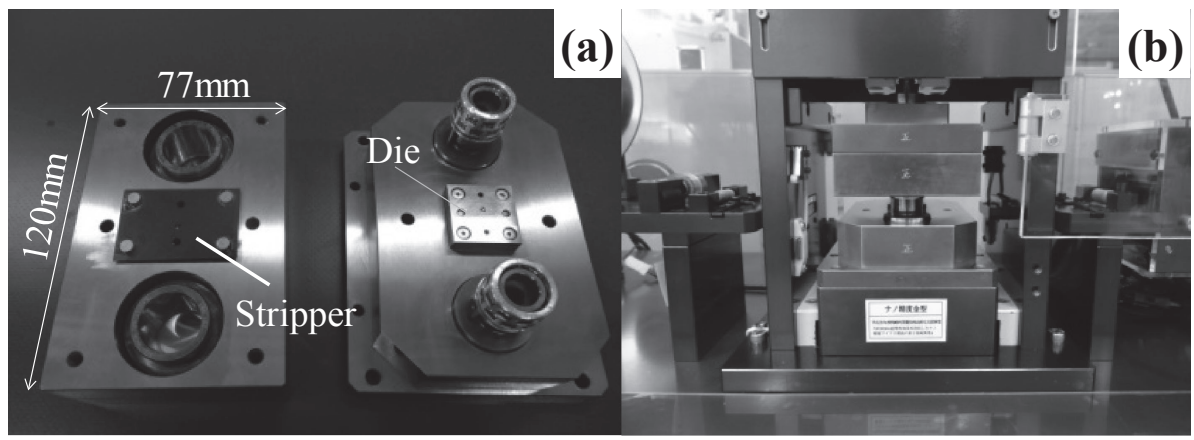

Fig. 3 Micro-shaping process to make micro-piercing and micro-embossing of bare stainless sheets and to fabricate the sheet elements. (a) A cassette die set, and (b) CNC micro-stamper.
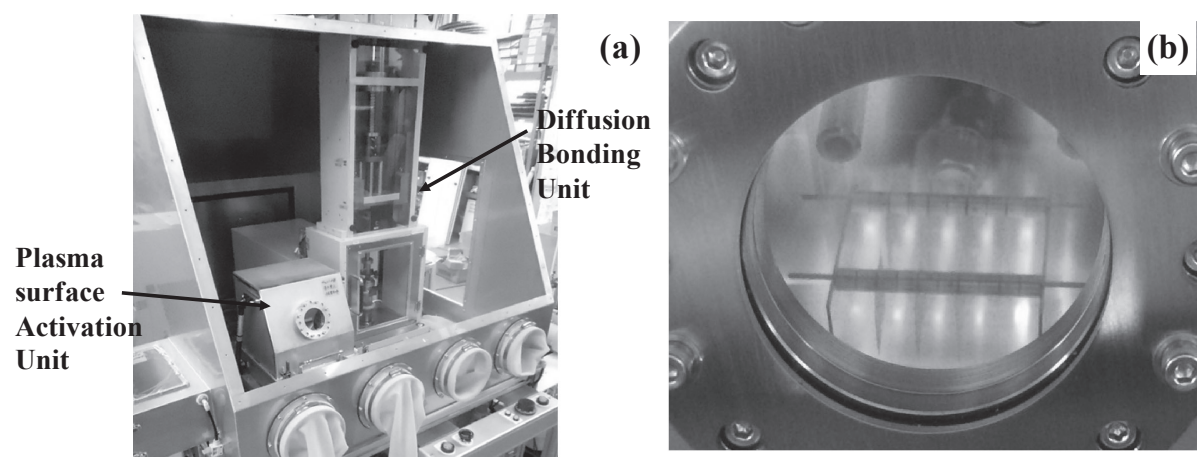

Fig. 4 Micro-joining system for integration of sheet elements into a product. (a) A micro-joining system working in the inert atmosphere, and (b) argon-hydrogen plasma surface activation process under the pressure of $80 \mathrm{~Pa}$.


Fig. 5 Micro-piercing of a bare AISI304 stainless steel sheet with the thickness of $10 \mu \mathrm{m}$. (a) A plasma-printed punch with three complex shaped heads and (b) micro-pierced sheet element with a circular pendulum and three S-lettered springs to be working as a valve.

\section{Experimental Results}

The plasma printing as well as the micro-piercing processes are first explained in the top-to-down procedure to yield the stainless steel sheet element with functional geometry by fine piercing with use of each plasma printed die pair. The plasma surface activation as well as uniaxial hot joining processes are next described as the down-to-top stream to integrate an assembly of sheet elements into a micro-pump.

\subsection{Plasma printing of micro-piercing punch and die}

AISI420 martensitic stainless steel substrate was prepared for plasma printing. After the procedure in Fig. 2, the micropunch for piercing the work sheets was fabricated as shown in Fig. 5(a). This piercing punch has three heads with the height of $80 \mu \mathrm{m}$ to shape a circular pendulum and three Sshaped springs into the sheet. The geometric model in Fig. 2 corresponds to this plasma printed punch in Fig. 5(a). This proves that every geometric model $P i$ for $1<i<n$ is accurately put into the i-th actual piercing punch.

The die core was also fabricated by projecting the above punch. After shaping and polishing, both the punch and coredie are set up into the cassette die-set pair. One cassette die set is fixed to the upper bolster in the CNC stamper while the other, to the lower bolster. After polishing the punch head and die surface, their edge has a curvature of $3 \mu \mathrm{m}$.

\subsection{Micro-piercing of sheet element}

The micro-piercing experiment was performed to yield a shaped sheet element. AISI304 sheet with the thickness of $10 \mu \mathrm{m}$ was utilized as a work material. As depicted in 




Fig. 6 Argon-hydrogen plasma surface activation of the pierced sheet elements. (a) Chemically processed stainless sheet surface, (b) plasma surface activated stainless steel surface, and (c) comparison of surface roughness between chemically and plasma activated sheets.
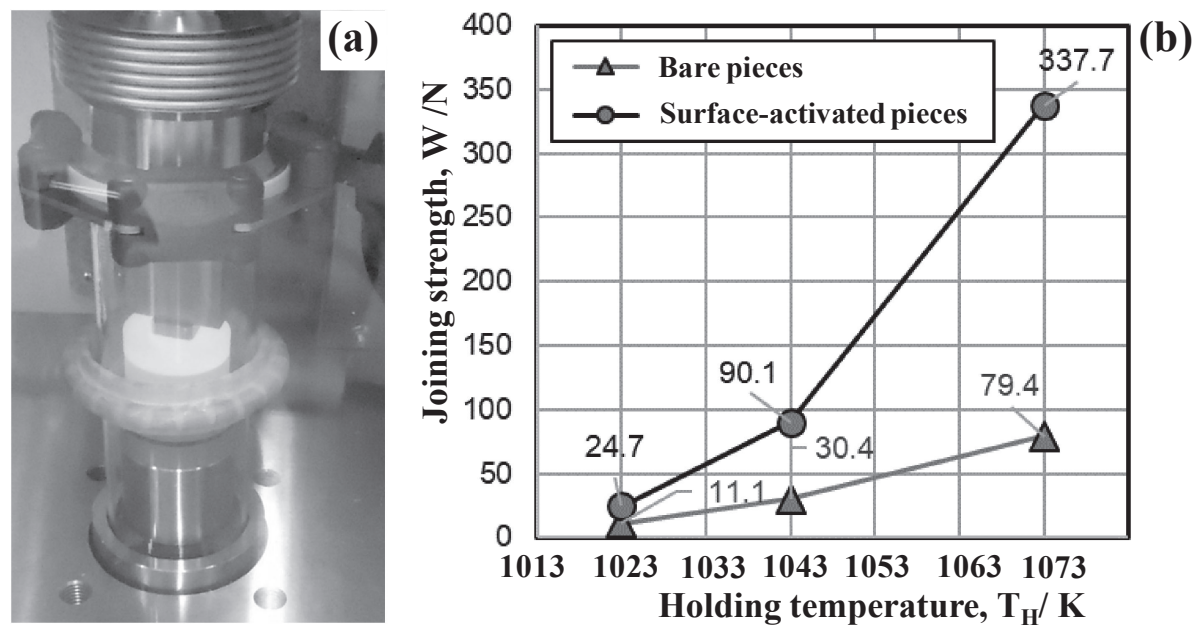

Fig. 7 Intelligent IH hot joining. (a) Uniaxial hot-joining experiment in the inert atmosphere, and (b) relationship between the measured joining strength by the peeling test and the average holding temperature.

Fig. 5(b), three holes are sheared out to leave a miniature valve unit in the stainless steel element. As tailored in Fig. 2(a), the pierced sheet has a circular pendulum with three S-shaped springs in practice. In the present fine piercing with nearly zero clearance between the plasma printed punch and die, less burrs as well as less fractured surface ratio in shearing are attained to yield the precisely sheared channels through each sheet element as proven in Fig. 5(b).

\subsection{Plasma surface activation}

The plasma surface activation is responsible for low temperature surface cleaning and polishing to reduce the passive film thickness for joining of shaped stainless steel sheet assembly in shorter takt time without damage to each element. Argon plasma is used to make physical surface treatment by ion bombardment effect; while, hydrogen plasma is employed to make chemical surface treatment by reduction reaction. The ratio of hydrogen to argon in the mixture gas must be optimized to make full use of the plasma surface activation. In the present study, this ratio is selected to be $20 \%$ from the in situ plasma diagnosis during the plasma surface treatment to be discussed.

The plasma activated AISI304 sheet surface is compared to the $\mathrm{NH}_{4} \mathrm{~F}$-processed one at the same time and temperature conditions to describe how much the stainless steel surface layer is reduced and polished. As compared between Figs. 6(a) and (b), the present plasma activation attains more smooth and thinner passive film layer than the chemical treatment. ${ }^{19)}$ In particular, the surface roughness measure- ment in Fig. 6(c) reveals the oxide passive film thickness is significantly reduced by the present plasma surface activation.

\subsection{Uniaxial hot joining}

The assembly of plasma surface-activated stainless steel elements is restacked into a cell unit in the inert atmosphere and uniaxially loaded for hot joining at the specified temperature. Figure 7(a) depicts a uniaxially hot-joining experiment with use of IH (Induction Heating) device. This device is designed to equip for two functions; 1) heating and rapid-quenching the upper and lower self-heating plates directly for uniform heating in lateral direction, and, 2) moving the IH-heating stage for uniform heating in the axial direction of stack. Owing to these features, the local-spot temperature in the inside of any sheet elements is homogenously fixed into a tolerance around the specified holding temperature.

The average holding temperature as well as the takt time for hot-joining are designed by the interfacial strength of joined unit via the peeling test. Figure 7(b) compares the relationship between the measured joining strength and the holding temperature with and without the plasma surface activation. When hot joining the bare stainless steel sheets without plasma surface activation at $1073 \mathrm{~K}$ (or $800^{\circ} \mathrm{C}$ ) for $1.8 \mathrm{ks}$ by $10 \mathrm{MPa}$, the joining strength is much lower than $100 \mathrm{~N}$. This joined unit has porous interfaces between neighboring sheet elements with the void ratio more than $60 \%$ and having a brittle fractography after peeling test. 


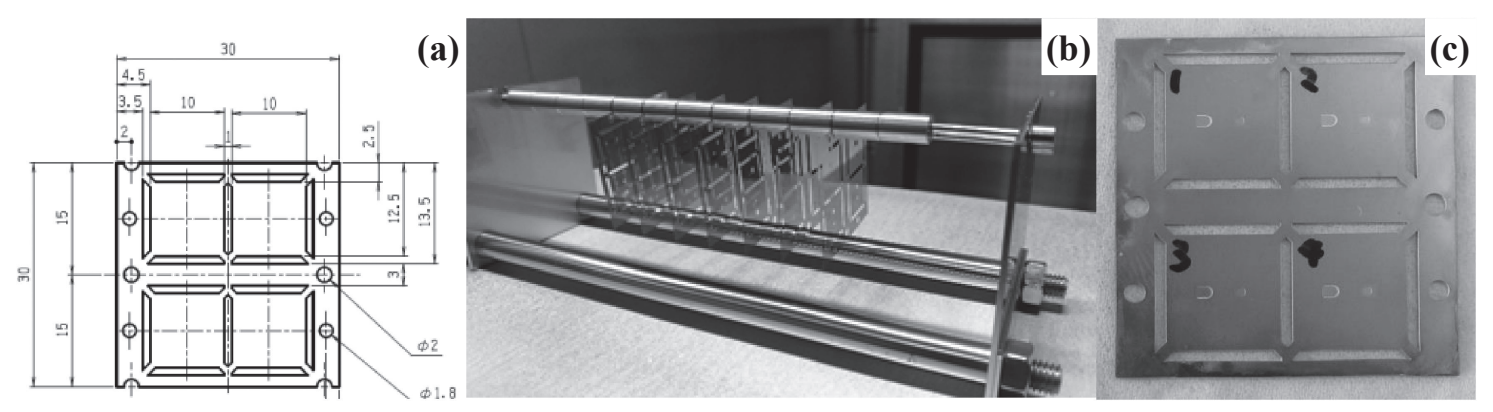

Fig. 8 Top-to-down and down-to-top procedures in the additive sheet-manufacturing from the original CAD to the final product. (a) CAD data for cold-stamped sheet elements, (b) laterally aligned stack of AISI304 stainless steel sheet elements, and (c) final joined product as four micro-pumps.

After hot joining the plasma surface activated AISI304 sheets at $1043 \mathrm{~K}$ (or $770^{\circ} \mathrm{C}$ ), the joining strength becomes close to $100 \mathrm{~N}$, where the void ratio becomes less than $50 \%$ in the fractography. Optimization of the self-heating plates by $\mathrm{IH}$ as well as the plasma conditions, can afford to shift this relationship to the lower holding temperature side. When changing the alumina jig to TiAlN coated Inconel one, the holding temperature to attain the joining strength of $100 \mathrm{~N}$ for $1.8 \mathrm{ks}$ by $10 \mathrm{MPa}$ is reduced down to $953 \mathrm{~K}$. This condition is selected as a standard uniaxial hot joining procedure in the following experiment.

\subsection{Fabrication of micro-pump by the additive sheet manufacturing}

Top-to-down and down-to-top procedures in the present method is summarized into Fig. 8. CAD data for each sheet element are prepared as a skeleton sheet format for cold stamping to have $\mathrm{n} \times \mathrm{n}$ micropumps into a unit cell; e.g., four base plates for $2 \times 2$ micro-pumps on the blank stainless steel sheet were designed in Fig. 8(a). The positioning holes were also included in CAD data together with four plate unit design.

In the top-to-down procedure as stated, each AISI304 sheet element is shaped to have a tailored texture and guild holes after this CAD data by cold piercing process with use of each plasma printed micro-die set. The down-to-top procedure starts from stacking the constituent sheet elements into an assembly in lateral, as depicted in Fig. 8(b). This lateral stack is surface-activated to clean and activate each surface of eight element sheets. This plasma treated stack is reassembled into the vertical stack of eight element sheets. This assembly is set in the fixture for uniaxial hot joining. After joining at $953 \mathrm{~K}$ for $1.8 \mathrm{ks}$ by $10 \mathrm{MPa}$, the joined stack is taken out from the chamber.

Figure 8(c) depicts the hot joined assembly of eight element sheets. This assembly is free from thermal distortion and irregular alignment by reducing the holding temperature below $973 \mathrm{~K}$ (or $700^{\circ} \mathrm{C}$ ) in the present joining procedure. This experiment proves that micro-pump arrays are microformed by the present additive sheet manufacturing.

\section{Discussion}

In the conventional top-to-down approach to produce the micro-pump, the chemical etching was utilized to perforate the channels into each element. Geometric irregularities often occurred at the edge of channels by over-etching behavior. The etching duration time was prolonged for thick sheets; the tailored blank sheets were difficult to use in the chemical etching. In the present case, each blank sheet is precisely sheared to form the channeled element sheet by fine piercing with use of the plasma printed punch and die. In addition, the sheared faces are fine and smooth as shown in Fig. 5(b). Furthermore, the tailored blank sheet is also shaped by fine piercing to have more complex channel geometry into element sheet. This implies that fine piercing approach with use of the plasma oriented die-set has much superiority to the chemical etching process for the top-to-down procedure of additive sheet-manufacturing.

Without use of the plasma surface activation, higher holding temperature than $1223 \mathrm{~K}$ (or $950^{\circ} \mathrm{C}$ ) is necessary to make oxygen and carbon diffusion into stainless steel matrix for bonding, as reported in Ref. 20). Even when using the hot joining, longer duration time than $7.2 \mathrm{ks}$ is needed to fabricate the joined assemblies in mass production. During the heating and cooling durations, the unjoined parts in assembly expand and shrink freely without constrains to enhance the thermal distortion and bending. This geometric irregularity deteriorates the leak proof of micro-pump since it is difficult to be repaired in the joined assembly. This proves that lower temperature processing is indispensable to improve the final product quality by reducing the thermal distortion as well as residual strains as an optimum down-totop procedure.

The surface activation conditions must be optimized to reduce the holding temperature and duration time in hot joining. In the present system, argon-hydrogen plasmas are ignited and sustained separately between two adjacent element sheets for simultaneous surface activation as shown in Fig. 4(b). There are two directions to enhance the surface activation process; e.g., optimization of hydrogen to argon flow rate ratio and optimal control of spatial impedance for each separate plasma sheath.

In the former optimization as stated before, the emissive optical spectroscopy was used to make plasma diagnosis on the hydrogen and argon plasma states. The activated hydrogen atom is characterized by $\mathrm{H}_{\alpha}$-peak while the argon ion, by $\mathrm{Ar}^{+}$-peak in diagnosis. As demonstrated in Ref. 21), the former peak increases with surface activation by reduction of oxide passive films by hydrogen atoms. The latter $\mathrm{Ar}^{+}$-peak enhances with ion bombardment onto the oxide film. Hence, the ratio of $\mathrm{H}_{\alpha}$-peak intensity to $\mathrm{Ar}^{+}$-peak 
one becomes a parameter for optimization of plasma species contents. This ratio reaches to maximum when the applied hydrogen gas content ranges from 20 to $30 \%$ in the argonhydrogen mixture gas.

In the latter, the alignment configuration in lateral assembly in Fig. 8(a) as well as the impedance control in each unit of assembly, play an important role to increase the argon ion density as well as the electron density in the plasma sheath. If this local spatial impedance could be improved, the hydrogen content would be increased to activate the chemical reduction for more surface activation. Fine tuning in the plasma sheath state by plasma diagnosis leads to search for more optimum surface activation conditions to reduce the holding temperature without increase of the takt time, or, to reduce the hot-joining duration of $1.8 \mathrm{ks}$ in the present study.

In the micro-joining by the intelligent $\mathrm{IH}$ unit, the selfheating plate works as a device to make rapid heating of stainless steel sheets in homogeneous. When heating a stainless steel sheet directly by $\mathrm{IH}$, the vicinity to its edge is only heated but other parts remains to be not heated. This device has a texture with through-groove network to distribute the self-heating hot-spots uniformly by uniform penetration of high frequency electromagnetic waves into networked plate. This indirect $\mathrm{IH}$ of stacked assembly provides the best solution to make homogeneous joining by the diffusion process between adjacent stainless steel sheets.

As partially discussed in Ref. 22), the leak of water from this micro-pump was controlled to be negligibly small when the void volume fraction on each joined interface became less than $30 \%$, or, the unjoined sections were isolated on each interface. Both the holding temperature and hot-joining duration could be reduced even with preservation of this leak-free function in the micropump.

\section{Conclusion}

The additive sheet-manufacturing is suitable to fabrication of leak-free micro-pumps with high integrity and toughness. In its top-to-down procedure, each stainless steel element sheet is precisely pierced by using the plasma printed die set to have the tailored through-channel texture in correspondence to the sliced CAD data of original product model. The plasma printing is effective to fabricate the miniature die set by micro-patterning the same geometric model as the sliced CAD data onto the starting punch and die substrates. In the down-to-top procedure, an assembly of shaped element sheets is surface activated for $900 \mathrm{~s}$ under the argonhydrogen plasmas and jointed at $953 \mathrm{~K}$ for $1.8 \mathrm{ks}$ by $10 \mathrm{MPa}$ to yield the integrated micro-pump with the peeling strength of $100 \mathrm{~N}$.

Their quality in function is assured by the interfacial strength and the distortion-free assembly through the lower temperature joining. The present manufacturing is also responsible for mass production of micro-pumps. Shorter takt time as well as lower temperature history are a key to yield the reliable products with sufficiently accurate geometry. In particular, further reduction of holding temperature below $900 \mathrm{~K}$ is expected to take place by optimization of the argon-hydrogen plasma conditions.

The plasma printing provides an automatic and systematic procedure to fabricate the micro-piercing and microembossing dies for each element of assembly without loss of product design. Every sheet element can be produced by CNC stamping with a die-exchanger and built into an assembly of elements. This microforming process is further integrated by the present processing to produce a three dimensional mechanical element, device and system even in mass.

\section{Acknowledgements}

The authors would like to express their sincere gratitude to the late Mr. Y. Sugita for his collaboration with us in the related research and development. The present study was financially supported in part by JST-program, Japan from 2015 to 2018.

\section{REFERENCES}

1) K. Igor (ed.): Pump Handbook, 4th ed., (McGraw Hill, New York, 2007).

2) R. Mackay: http://www.pumpscout.com/articles-expert-advice (2019/ 7/20).

3) http://www.condorpumps.co.nz/Pumps/Cast-iron-pumps_2 (2019/7/20).

4) https://morganbronze.com/custom-machined/pump-valve/ (2019/7/20).

5) FDA: Infusion pumps total product life cycle, FDA-Guidance, (2014) pp. 1-36.

6) F. Flynn, L. Mohr and P. Lawlor-Klean: Jt. Comm. J. Qual. Saf. 29 (2003) $37-40$

7) https://www.boxerpumps.com/en/products/miniature-diaphragm-pumpsliquid/ (2019/7/20)

8) N.-T. Nguyen, X.Y. Huang and T.K. Chuan: J. Fluids Eng. 124 (2002) 384-392.

9) J. Zhang and Y.-G. Jung: Additive Manufacturing: Materials, Processes, Quantifications and Applications, (Butterworth-Heinemann, Oxford, 2018)

10) F. Lasagni: Proc. 22nd Int. ESAFORM Conf. Mater. Form. AIP Conf. Proc. 2113, (2019) 050143-1-6.

11) W.W. Wits, S.J. Weitkamp and J.v. Es: Procedia CIRP 7 (2013) 252257.

12) D. Baffari, G. Buffa, D. Companella, G. Ingarao, E.L. Valvo and L. Fratini: Proc. 22nd Int. ESAFORM Conf. Mater. Form. AIP Conf. Proc. 2113, (2019) 050214-1-6.

13) T. Aizawa, T. Satoh and T. Shiratori: Proc. 22nd Int. ESAFORM Conf. Mater. Form. AIP Conf. Proc. 2113, (2019) 050007-1-6.

14) T. Satoh, T. Aizawa, T. Shiratori, Y. Sugita and M. Anzai: Procedia Manuf. 15 (2018) 1475-1480.

15) T. Satoh, T. Aizawa, T. Shiratori and S.-I. Yoshihara, J. JSTP 60 (2019) 80-84.

16) T. Aizawa, T. Takashima and T. Shiratori: Proc. 8th AWMFT, (2015) pp. $17-22$.

17) T. Shiratori, T. Aizawa, Y. Saito and K. Wasa, Metals 9 (2019) 396.

18) T. Aizawa: Stainless Steels, Ch. 3, (IntechOpen, 2019) pp. 31-50.

19) L. Wong, T. Suratwala, M.D. Feit, P.E. Miller and R. Steele: J. NonCryst. Solids 355 (2009) 797-810.

20) O. Ohashi and S. Suga: J. Japan Inst. Metals 56 (1992) 579-585.

21) E.Y. Ersyzario: PhD Thesis, Shibaura Institute of Technology, (2016).

22) T. Aizawa and T. Shiratori: Proc. 12th AWMFT-2019, (2019) 1-6. 